\title{
A Technology Demonstrator for 1.6-2.0 THz Waveguide HEB Receiver with a Novel Mixer Layout
}

\author{
D. Dochev ${ }^{1}$, V. Desmaris, D. Meledin, A. Pavolotsky, V. Belitsky \\ Group for Advanced Receiver Development (GARD), \\ Department of Earth and Space Sciences, \\ Chalmers University of Technology, SE-412 96 Gothenburg, Sweden
}

\begin{abstract}
In this paper, we present our studies on a technology demonstrator for a balanced waveguide hot-electron bolometer (HEB) mixer operating in the $1.6-2.0 \mathrm{THz}$ band. The design employs a novel layout for the HEB mixer combining several key technologies: all-metal $\mathrm{THz}$ waveguide micromachining, ultra-thin $\mathrm{NbN}$ film deposition and a micromachining of a silicon-on-insulator (SOI) substrate to manufacture the HEB mixer. In this paper, we present a novel mixer layout that greatly facilitates handling and mounting of the mixer chip via self-aligning as well as provides easy electrical interfacing. In our opinion, this opens up a real prospective for building multi-pixel waveguide $\mathrm{THz}$ receivers. Such receivers could be of interest for SOFIA, possible follow up of the Herschel HIFI, and even for ground based telescopes yet over limited periods of time with extremely dry weather (PWV less than $0.1 \mathrm{~mm}$ ).
\end{abstract}

Keywords hot electron bolometer (HEB) mixer, micromachining, radio astronomy instrumentation, silicon-on-insulator, terahertz waveguide.

\section{INTRODUCTION}

An airborne observatory such as SOFIA [1], or a space observatories as Herschel [2] and Plank [3] allow to reduce or eliminate atmospheric absorption for observations in the terahertz frequency band. Moreover, high altitude and dry sites such as Dome $\mathrm{C}$ in Antarctica [4], Llano de Chajnantor [5, 6] and Cerro Chajnantor [7] in Chile could provide reasonable atmospheric transmission at $\mathrm{THz}$ frequencies thus making them suitable for technology testing and radio astronomical observations from the ground $[5,8]$ over limited periods of time when PWV is less than $0.1 \mathrm{~mm}$.

\footnotetext{
${ }^{1}$ Corresponding author Dimitar Dochev (dimitar.dochev@ chalmers.se, tel. +46 317721850 , fax +46 317721850 ).
} 
Heterodyne instrumentation for the THz ground - based telescopes, airborne and space observatories has made remarkable progress during the last two decades bringing SIS and HEB mixer technology to its ultimate state [9-11]. Often above $1 \mathrm{THz}$ a quasioptical HEB mixer design with planar antenna and substrate lens $[12,13]$ is used. Alternatively, waveguide HEB mixers have been also demonstrated $[5,8,14]$ offering superior efficiency of the receiver - antenna coupling via a corrugated horn. Furthermore, waveguide designs present a natural filtering of the out-of-the-band background noise, which is especially important for easily saturated HEB mixers. When installed at the ground-based telescopes, the atmospheric contribution to the background noise remains close to $300 \mathrm{~K}$ at terahertz frequencies and could cause saturation. Additionally, $\mathrm{THz}$ receivers have to cope with the relatively low attainable power of local oscillator (LO) sources and its sufficiently high sideband noise [15]. These problems could be circumvented by employing a balanced layout, which offers extremely high efficiency of using the LO power (around $3 \mathrm{~dB}$ loss only) and provides improved receiver stability by intrinsic cancellation of the LO amplitude noise as it was demonstrated in [5].

The dimensions of all components are scaled down with the frequency and for frequencies, e.g., around $1.3 \mathrm{THz}$ the waveguide dimensions are $90 \times 180 \mu \mathrm{m}$ [2] and required even smaller nearly sub-micron design tolerances. This introduces increasing challenges for fabrication using conventional machining. In this work, we present a technology demonstrator of a waveguide balanced HEB mixer for $1.6-2.0 \mathrm{THz}$ band, representing a step-up in frequency for micro machining technology for terahertz all-metal waveguides [16, 17]. Additionally, we investigate possibilities for a simplified and accurate mounting, and electrical interfacing of the HEB mixer substrate. The mixer employs a splitblock waveguide housing and utilizes a novel HEB mixer chip layout.

\section{MIXER DESIGN}

\subsection{Mixer layout}

Similar to [5], we have chosen to use a balanced waveguide HEB mixer layout. However, differently to the mixer presented in [5] that uses the split-block and back-piece layouts combined, the current design employs the split-block configuration for the whole mixer block. The mixer layout, presented in Fig. 1, consists of input waveguides connecting to the corrugated horns (for RF and LO), a waveguide $3-\mathrm{dB} 90^{\circ}$ hybrid with its outputs connected to two HEB mixers.

In the split-block design, the length for the RF signal waveguides could be minimized reducing the RF losses by placing the RF $3-\mathrm{dB} 90^{\circ}$ hybrid input at minimum possible distance to the corrugated horn and introducing the HEB 
mixer chips of the balanced mixer directly at the hybrid outputs as compared to the balanced mixer presented in [5]. When the mixer housing is fabricated using the split-block technique, the corrugated horns are inserted in the dedicated recesses after joining the mixer block split halves and thereafter the horns are aligned with the input waveguides.

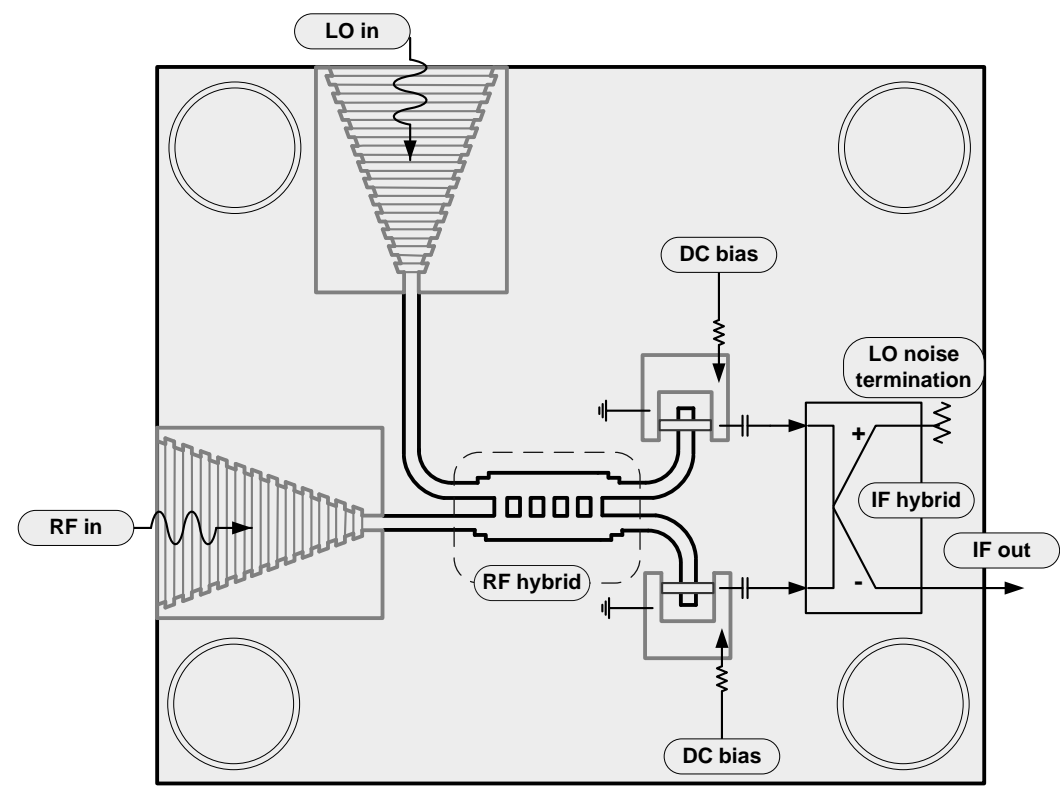

Fig. 1 Proposed schematic mixer layout. The balanced waveguide mixer consists of corrugated horns (for RF and LO) connected to a waveguide $3 \mathrm{~dB} 90^{\circ}$ hybrid with its outputs connected to two HEB mixers. The HEB mixers employ a novel layout with supporting $\Pi$-frame. The waveguide full cross-section dimensions $60 \times 120 \mu \mathrm{m}, 1.6-2 \mathrm{THz}$ band.

\subsection{Waveguide $3 \mathrm{~dB} 90^{\circ}$ hybrid}

The input 3-dB waveguide hybrid provides the LO injection and RF signal distribution between the two HEB mixers with a $90^{\circ}$ phase shift. In general, $90^{\circ}$ hybrids offer substantially wider RF band as compared to $180^{\circ}$ RF hybrid option [18]. In the proposed design, the hybrid is implemented as a waveguide branchline coupler and consists of five sections (Fig. 2) in order to ensure the required performance across the frequency band. The chosen type of the hybrid offers an advantage of having larger branch heights and thus simplifying the hybrid fabrication [19]. 


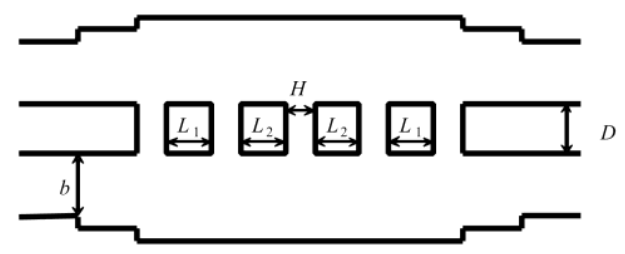

Fig. 2 Cross-section of the $3-\mathrm{dB} 90^{\circ} \mathrm{RF}$ hybrid $\left(H=22 \mu \mathrm{m}, L_{1}=33 \mu \mathrm{m}, L_{2}=31 \mu \mathrm{m}\right.$, $D=44 \mu \mathrm{m}$ and $\Delta b=6 \mu \mathrm{m})$. Dimensions are optimized using 3D EM simulation software.

A single-mode full-height waveguide for the frequency band of 1.6-2.0 THz has dimensions of $60 \times 120 \mu \mathrm{m}$. The hybrid is optimized using a 3-D electromagnetic (EM) simulation packages $[20,21]$. The design variables used in the optimization procedure are: the spacing between branches $\left(L_{\mathrm{n}}\right)$, the height of the branches $\left(H_{\mathrm{n}}\right)$ and the distance between the main waveguides $(D)$. Figure 3 shows the simulated performance of the hybrid. The return loss and the isolation between the hybrid input ports are expected to be better than $-20 \mathrm{~dB}$, while the amplitude and phase imbalance should be better than $\pm 1.5 \mathrm{~dB}$ and $90^{\circ} \pm 1^{\circ}$, respectively.
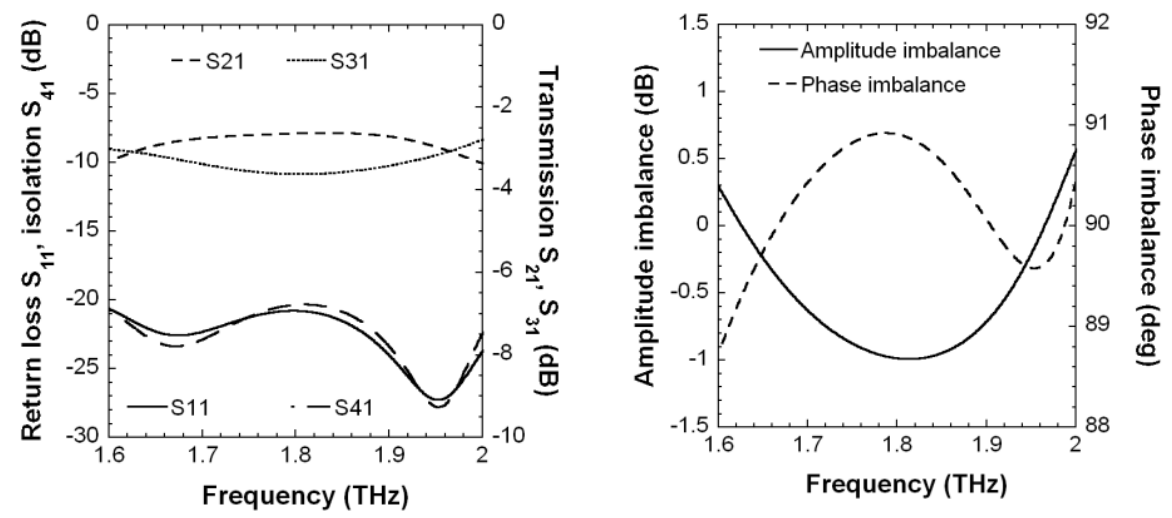

Fig. 3 Simulation results of the five-branch waveguide quadrature hybrid as illustrated in Fig. 2

\subsection{HEB mixer RF design}

Most of the SIS and HEB waveguide mixers utilize planar E-field probes in order to couple the waveguide EM field to a planar structure of the mixer on the substrate produced by means of thin-film technology. As discussed above in section 2.1, we opted for the split-block technique. The advantages of this design is the use of a non-radiating split of the waveguide, convenience of mounting of the active component and making all necessary DC and IF connections in one of the halves. 
Clearly, it is important to design the E-probe in such way that the incoming waveguide mode is coupled to the hot-electron bolometer mixer in a highlyefficient way. We have used a radial planar E-probe extensively discussed in $[22,23]$. However, the basic probe configuration was changed as suggested in [24], where the RF probe is connected on its arc-side to another port via a high impedance line, Fig. 4, in order to allow the mixer substrate to cross the entire full-height waveguide and provide the RF isolated port for the DC/IF as discussed in $[24,25]$.

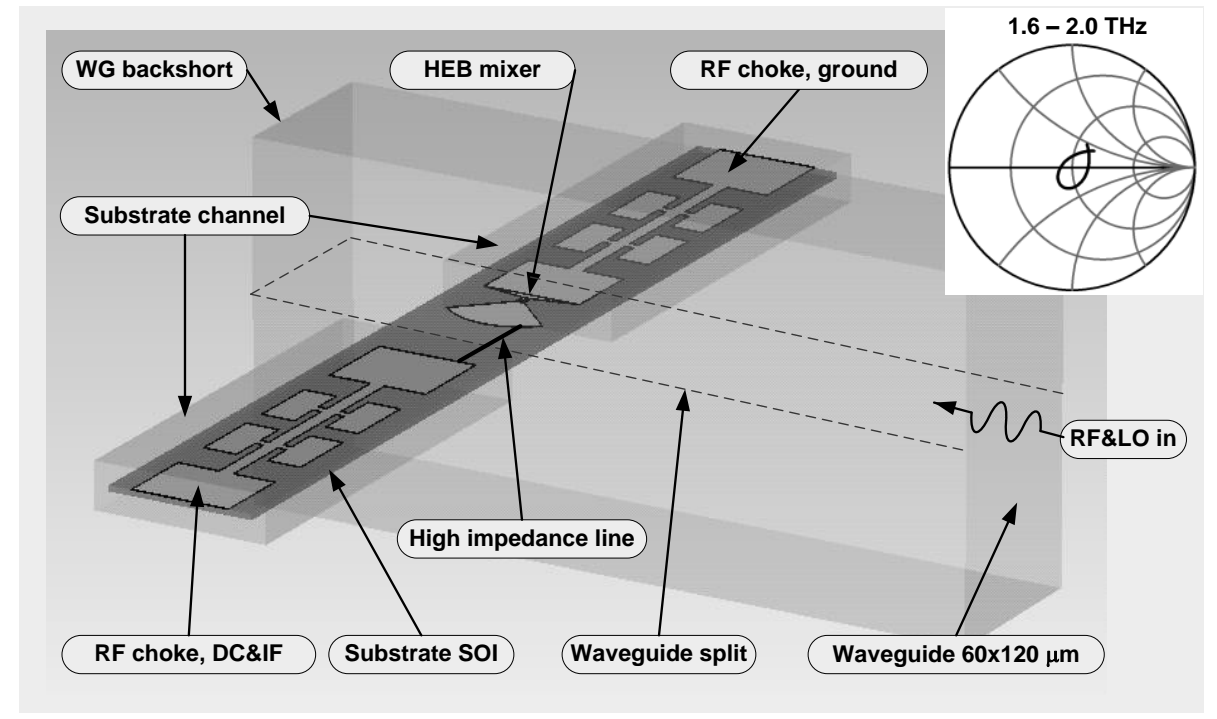

Fig. 4 The substrate RF layout: waveguide-to-substrate transition employing E-probe [24]. Insert shows the simulation results (Smith chart) of the radial RF probe on a $360 \times 50 \times 2 \mu \mathrm{m} \mathrm{Si}$ substrate for probe impedance of $50 \Omega$.

In order to define the dimensions and the critical margins of the design, we have performed 3D electromagnetic simulations using 3D EM simulation software $[20,21]$. For the optimized design, the silicon substrate has dimensions of $360 \times 50 \times 2 \mu \mathrm{m}$ and the probe output impedance is nearly pure real and close to $50 \Omega$ (insert, Fig. 4). The choice of the substrate dimensions is defined by constrains of avoiding the substrate propagation mode accounting for a high dielectric permittivity of the $\mathrm{Si}, \square_{\mathrm{r}}=11.6$. Another important consideration is the substrate thermal conductivity providing necessary cooling for the HEB mixer [26, 27].

Waveguide mixers usually use choke filters to efficiently block the leakage of the RF signal into the substrate channel and to provide virtual RF ground for mixer components placed onto the substrate. The choke structure is used simultaneously for IF extraction and to supply a DC bias to the HEB mixer. The 
geometry of the hammer sections has been optimized to give the rejection better than $-40 \mathrm{~dB}$ in the $1.6-2.0 \mathrm{THz}$ band.

\subsection{HEB mixer chip layout}

In order to facilitate mounting and IF/DC connecting of the terahertz HEB mixer chip, we propose a novel layout. The mixer substrate described in the section 2.3 has dimensions of $360 \times 50 \times 2 \mu \mathrm{m}$ and clearly would be extremely difficult to manipulate and integrate in the mixer block. Earlier suggested beamleads and membrane solutions [28-30] partly solve the problem of the electrical interfacing but do not provide improved handling by leaving the substrate size as small as it is. Another proposed solution employs a micro - machined frame supporting the mixer substrate $[31,32]$, providing far more possibilities to handle the mixer chip. However this type of design requires a back-piece configuration making it incompatible with the split-block technique and most likely will not be suitable for $\mathrm{THz}$ multi-pixel receiver applications because of the back-piece mechanical dimensions will conflict with the optics defined pixel pitch.

We suggest a different layout that uses a half-frame supporting the mixer substrate, as outlined in Fig. 5. The mixer substrate is made as a beam and contains the HEB device, RF chokes, an E-field probe and a high-impedance line. A П-shaped bulk silicon frame is left around the beam in order to ease the handling and electrical contacting. The shape and the dimensions of the supporting $\Pi$-frame are chosen such that it provides alignment reference with respect to the corresponding recess in the mixer housing where it should be integrated.

The shape of the HEB mixer chip is defined via micromachining, photolithography and consequent etching, as described in Section 3.1, while the thickness of the beam and the supporting frame depends on the SOI substrate. Consequently, in order to integrate the HEB mixer chips having this novel layout into the mixer block no additional lapping and dicing steps are necessary.

Before starting the actual fabrication, we have investigated the mechanical behavior of the structure, subjected to a temperature change from room temperature $(300 \mathrm{~K})$ down to $4.2 \mathrm{~K}$, by performing thermo-mechanical simulations using commercial finite element model (FEM) software [33]. The predicted total deformation of the beam under cooling results to less than $1 \mathrm{~nm}$ with corresponding maximum principal stress of $15 \mathrm{MPa}$, which is about $2 \%$ of the ideal shear strength value for Si [34]. Moreover, the individual devices have been DC measured at $4.2 \mathrm{~K}$ and no fractures or deformations of the tested structures have been observed. 


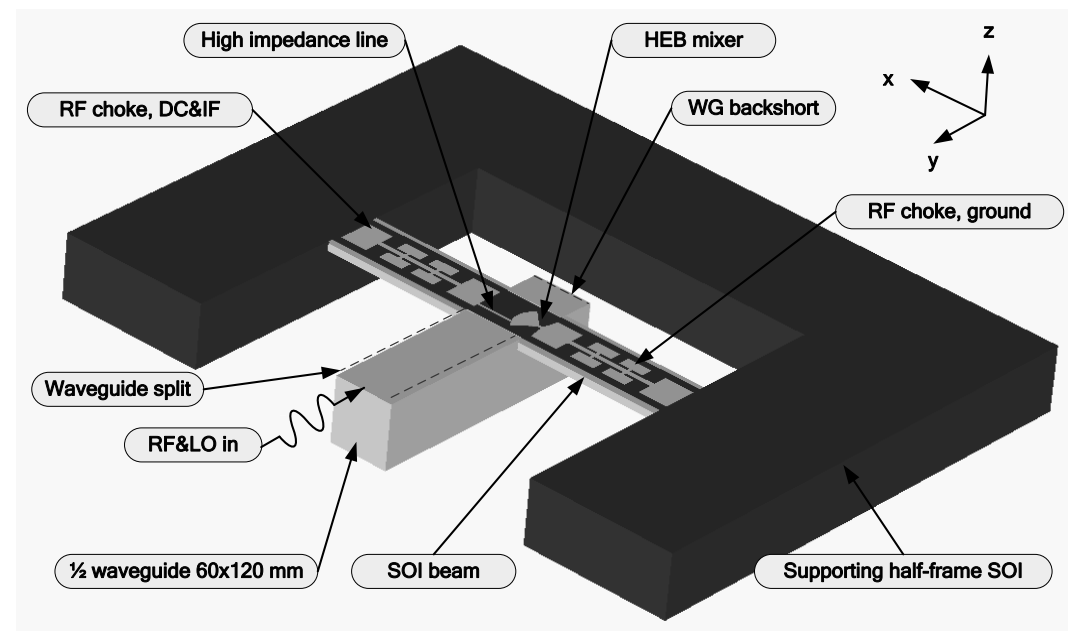

Fig. 5 An illustration of the HEB mixer chip with supporting half-frame. This novel layout is fully compatible with the split-block technique and should dramatically ease mounting and electrical contacting to the mixer chip allowing e.g. a wire-bonding to the contact pads (not shown) deposited on the beam supporting frame.

\section{MIXER FABRICATION}

\subsection{Mixer chip}

The hot-electron bolometer (HEB) devices were fabricated on a $14 \times 14 \mathrm{~mm}^{2}$ double-bonded SOI substrate [35]. The $\mathrm{NbN}$ films were grown on the preheated $\left(\sim 700^{\circ} \mathrm{C}\right)$ substrates by means of reactive DC magnetron sputtering in a $\mathrm{Ar} / \mathrm{N}_{2}$ gas mixture using a $2 " \mathrm{Nb}$ target, resulting in the $\mathrm{NbN}$ film deposition rate $75 \AA /$ min calibrated via the transmission electron microscopy (TEM) on satellite $\mathrm{NbN} / \mathrm{Si}$ specimens. The critical temperature of the deposited film is $9.2 \mathrm{~K}$.

The bolometer was defined by the separation between its contact pads, ranging from $100 \mathrm{~nm}$ to $400 \mathrm{~nm}$ [36]. Definition of the $\mathrm{NbN}$ bridge between the contact pads was accomplished by a reactive ion etching in $\mathrm{CF}_{4} / \mathrm{O}_{2}$ gas mixture through a mask defined in e-beam negative resist. After etching, the negative resist mask was left on top of the HEB bridge as a protection against atmospheric degradation factors. The contact pads were patterned by electron-beam lithography using a bilayer PMMA/Copolymer resist system. Ti/Au (3/30 nm) contacts were then evaporated followed by a lift-off. A consequent evaporation and lift-off steps were used to pattern the Ti/Au filter strictures. 


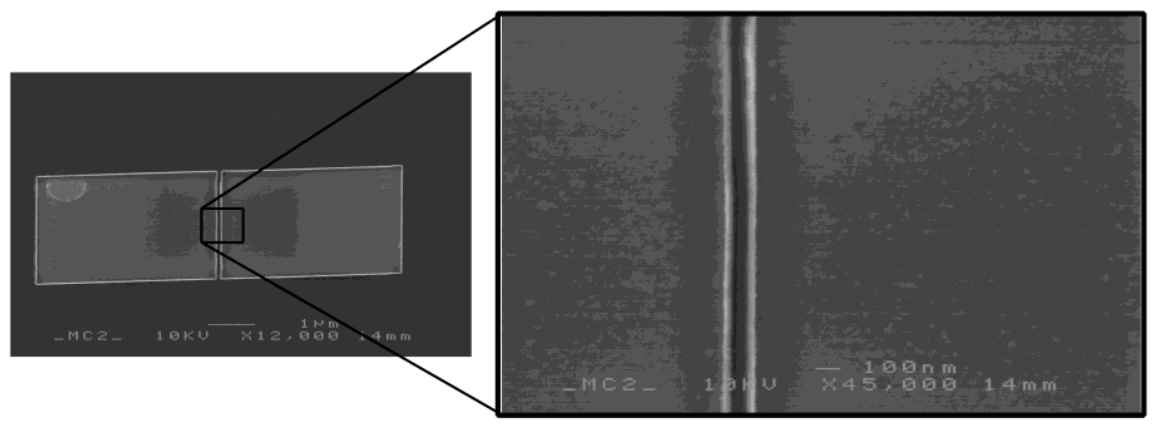

Fig. 6 SEM image of HEB contact pads after lift-off with a close-up view of the gap between the contact pads defining the length of the HEB mixer $\sim 100 \mathrm{~nm}$.

The fabrication procedure of the membrane beam structure, which carried the HEB RF circuitry, utilizes SOI substrate consisting of five layers, as could be viewed in Fig. 7a. The beam is defined on the front side of the wafer by photolithography and subsequent deep reactive ion etching (DRIE) Bosch process [37]. Having the wafer mounted face-down onto the carrier wafer, the bulk silicon holder layer is etched down till the oxide etch-stop layer is reached. The HEB support frame is consequently defined by another DRIE/Bosch process step. After that, the second $\mathrm{SiO}_{2}$ etch stop layer was removed by dry etching, see Fig. 7c. The beam with its HEB devices and frame are now completely defined and released from the carrier wafer, Fig. $7 \mathrm{~d}$. Note that the described process does not require any dicing step and all dimensions have the accuracy of the photolithographic process. 


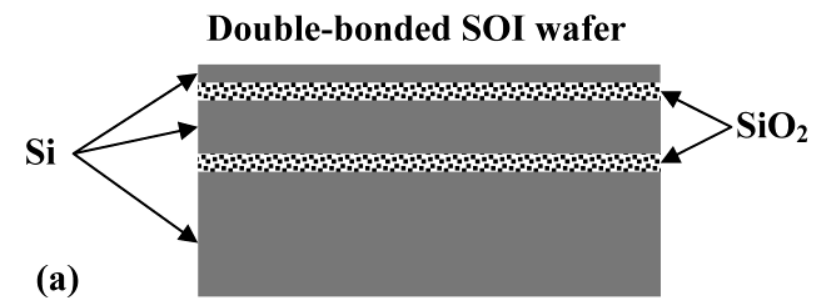

Wafer mounted upside-down on Pyrex carrier wafer

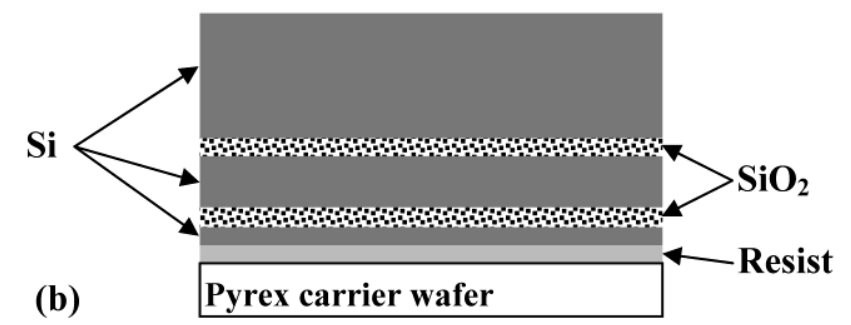

Wafer thinning and definition of support frame

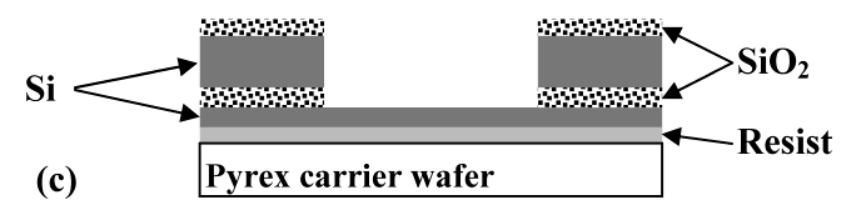

Free device and support frame

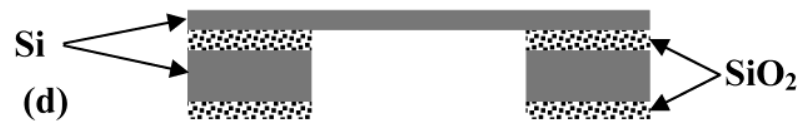

Fig. 7 Membrane beam fabrication steps. (a) Cross-section of the double-bonded SOI wafer. Layer pattern (from bottom): Si handle $(300 \mu \mathrm{m})$, oxide etch stop layer $(2 \mu \mathrm{m})$, high-resistivity Si layer $(57 \mu \mathrm{m})$, oxide etch stop layer $(0.5 \mu \mathrm{m})$ and high-resistivity Si device layer $(2.5 \mu \mathrm{m})$. (b) The wafer is mounted upside down on a carrier wafer. (c) The bulk Si handle is stripped by DRIE process and the supporting frame is defined by subsequent backside alignment and a Bosch process. (d) The protection resist is dissolved and the individual are released from the carrier wafer.

SEM micrographs of the ready processed beam structure are presented in Fig. 8. 

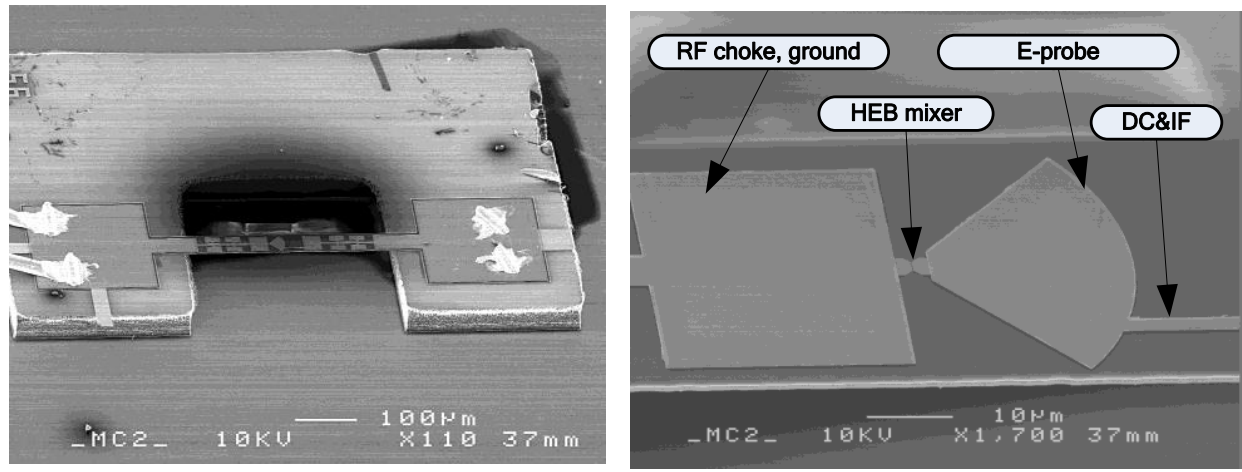

Fig. 8 SEM picture of the novel HEB mixer layout. Right: the half-frame provides solid support for the fragile beam while its shape facilitates mounting of the mixer into the split block. Left: detailed view of the E-probe and HEB mixer RF layout.

\subsection{Mixer block}

The mixer block has been manufactured following the all-metal waveguide micromachining technique (GARD-process) $[16,17]$. The SEM images of the fabricated mixer block are shown in Fig. 9.

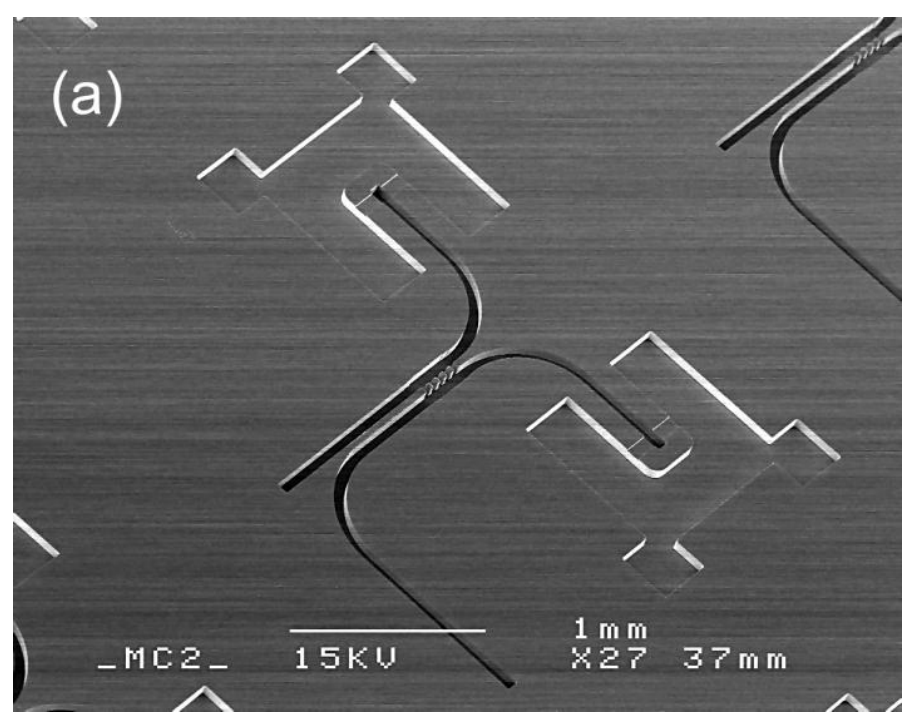



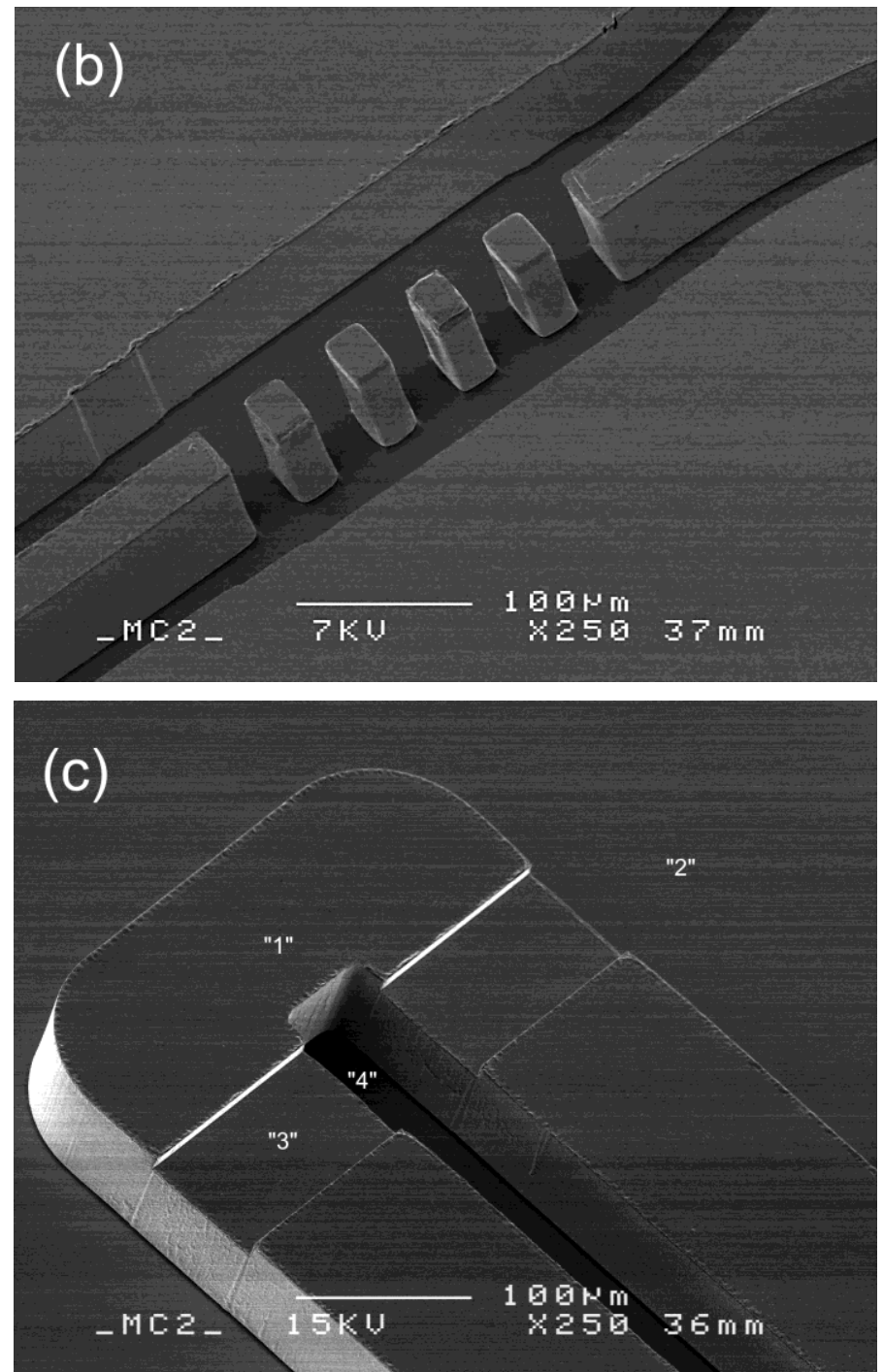

Fig. 9 SEM images of (a) the fabricated all-metal mixer block with waveguide dimensions $60 \times 120 \mu \mathrm{m}(60 \times 60 \mu \mathrm{m}$ in every split half). (b) Magnified view of the RF hybrid made as waveguide branch-line coupler with dimensions as in Fig. 2. (c) Magnified view of the substrate channel. Here "1", "2", "3" and "4" show the areas where the surface roughness measurements were performed (see discussion below and Fig. 10)

Surface roughness measurements of the split surface, substrate channel, waveguide bottom and the frame housing recess were performed using a Wyko NT1100 surface profiler and results are presented in Fig. 10. The RMS surface roughness is below $20 \mathrm{~nm}$ at all levels and it is about twice less than the estimated skin depth at $2.0 \mathrm{THz}$. 

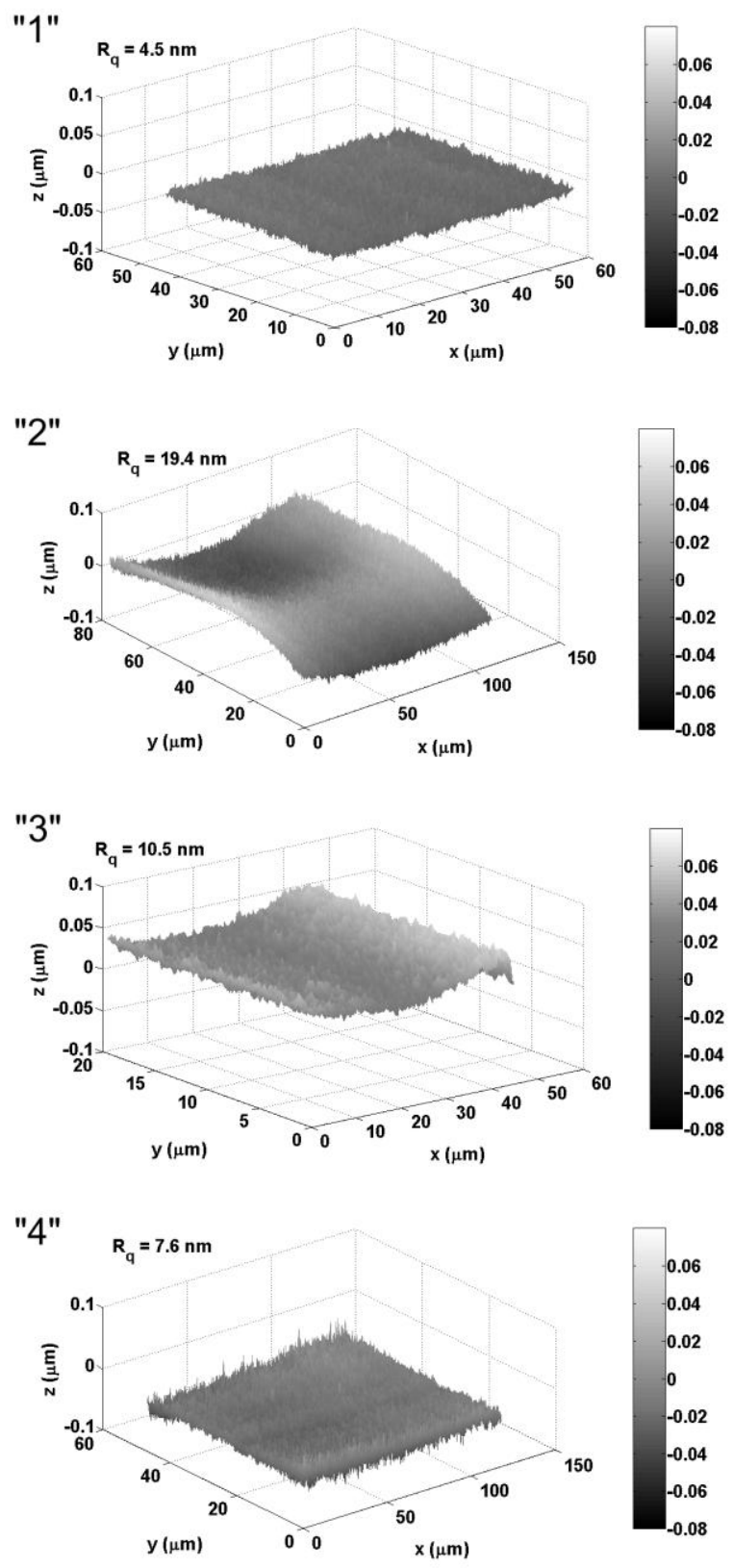

Fig. 10 Surface roughness measurements of the produced mixer block. $R_{\mathrm{q}}$ indicate the RMS roughness over the areas marked in Fig. 9c.

\subsection{Mixer chip DC test results}


In order to confirm that the suggested processing does not affect the HEB mixer performance, ideally we should perform RF tests of the HEB mixer with the novel layout. However, the purpose of our studies was technology demonstration. Besides, we are lacking suitable LO source covering the band of interest. Nevertheless, in order to have a proof for suitability of the new mixer layout and the required SOI substrate processing, we have chosen to measure critical current and critical temperature of ready devices. These parameters largely define the HEB mixer quality. The measurements confirmed acceptable change of the HEB fabricated devices as compared with the original device parameters before the beam processing. The fabricated wafer contained 70 devices whereas 15 of them were wired for DC testing. The spread of the measured critical current at $4 \mathrm{~K}$ and normal-state resistance at $20 \mathrm{~K}$ are $\pm 1.95 \mu \mathrm{A}$ and $\pm 2.7 \Omega$, respectively, in line with device uniformity data presented in [36]. This indicates suitability of the proposed process for fabrication of state-of-theart HEB mixers for balanced and multipixel array receivers. In Fig. 11, the resistance-temperature dependence - (a) and the IV-curves (b) of one of 70 fabricated devices are shown. Solid and dash lines illustrate the cases before and after the beam structure formation, respectively.

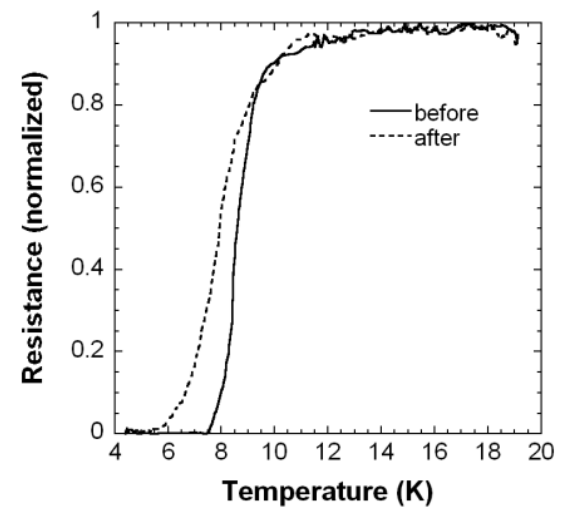

(a)

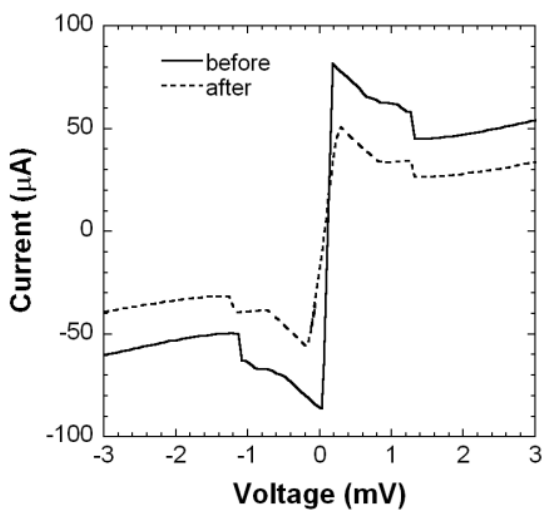

(b)

Fig. 11 Examples of measured (a) resistance-temperature dependence- and (b) current-voltage characteristics of the HEB devices before (solid) and after (dashed) beam structure fabrication

DC contact to the HEB chip was performed via wire bonding (Fig. 8). During our studies, the $\mathrm{NbN}$ films showed no indications of oxidation-based aging while the samples were processed about 1 month after the $\mathrm{NbN}$ film deposition. After processing, a slight decrease in the critical current compared to the initial tests before beam fabrication was observed, yet the critical temperature of the bolometers was barely affected by the process. This behavior could be attributed to the high hard-baking temperature, $\sim 200^{\circ} \mathrm{C}$, used for the protection resist layer. 


\subsection{Mixer integration}

The mixers were integrated into the mixer block as shown on Fig. 12. Our experience shows that the presented mixer layout greatly facilitates mounting and aligning of the HEB devices in the mixer block. Alignment relies on photolithography defined dimensions of the HEB beam, frame and the mixer block structure. Through this, the entire mounting procedure becomes self-aligning.
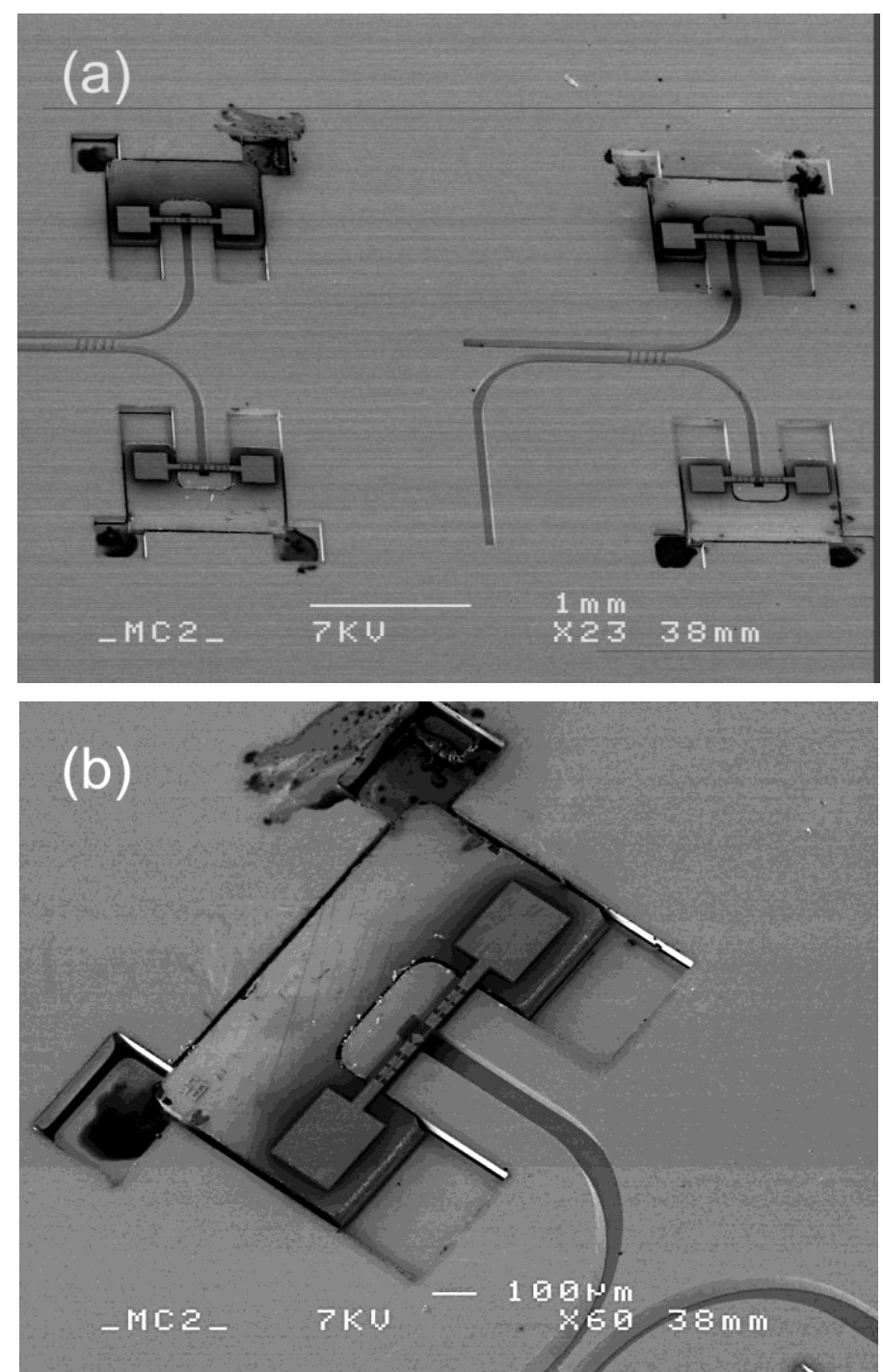


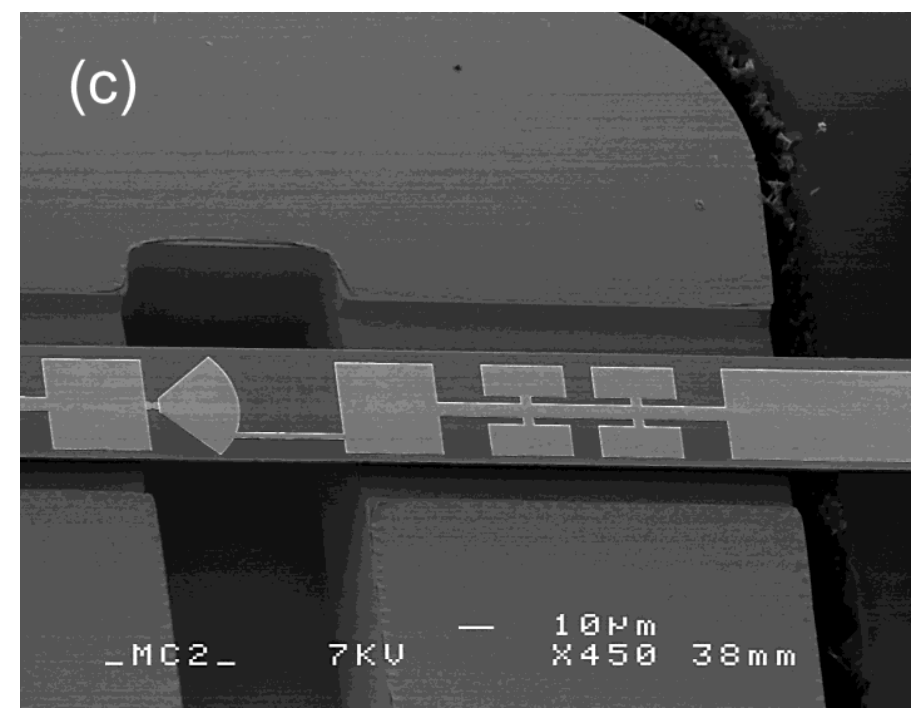

Fig. 12 SEM pictures of the fabricated mixer with integrated HEB mixer chips. No wire bonding for DC/IF interfacing was made at this stage. Magnified views of (b) a single HEB device and (c) HEB mixer beam crossing the waveguide.

It is worth mentioning that the GARD-process, used for the fabrication of allmetal waveguide structure, is inherently a lithographic process with accuracy of less than $1 \mu \mathrm{m}$, and hence can replicate identical waveguide mixer block structures over a 4" wafer. Its combination with the novel type of HEB chips provides high handling and alignment possibilities and opens the way for realization of multi-pixel $\mathrm{THz}$ waveguide receivers.

An important consideration for the mixer design and assembly is the sensitivity of the performance to a misalignment of the beam including the patterned RF circuitry, the chokes and the probe, with respect to the substrate channel and the main waveguide. We have performed 3D EM simulations in order to better understand the effects of different misalignments on the mixer input matching over the frequency band of interest.

Figure 13 shows the input matching dependence on the beam misalignment when the RF circuitry structure is perfectly centred to the beam. The position of the beam is varied from its nominal position inside the channel along Y-axis, with the coordinate system presented in Fig.5, by $\pm 2 \mu \mathrm{m}$ (Fig. 13a) causing insignificant changes in the performance. More considerable changes occur when the probe is misaligned with respect to main waveguide, along $\mathrm{X}$ direction by $\pm 2 \mu \mathrm{m}$ (Fig. 13b). We have chosen $\mathrm{X}$ and $\mathrm{Y}$ variation range, following to the real mounting accuracy achieved according to the geometry measurements performed via SEM studies over a number of the installed chips. 
We believe that the sensitivity of the probe performance to the $\mathrm{X}$-axis misalignment could be further reduced by refinement in the RF design.

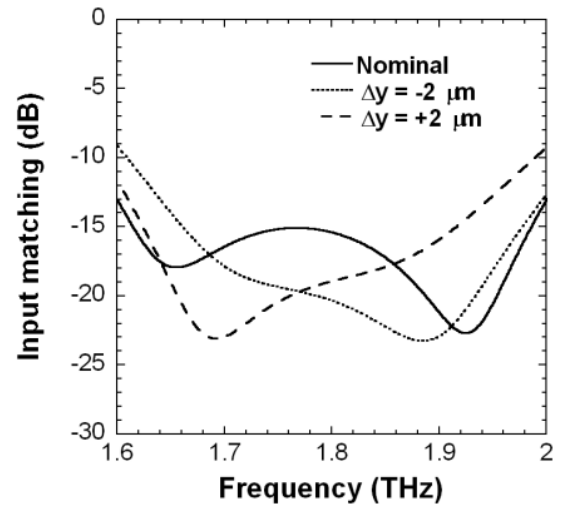

(a)

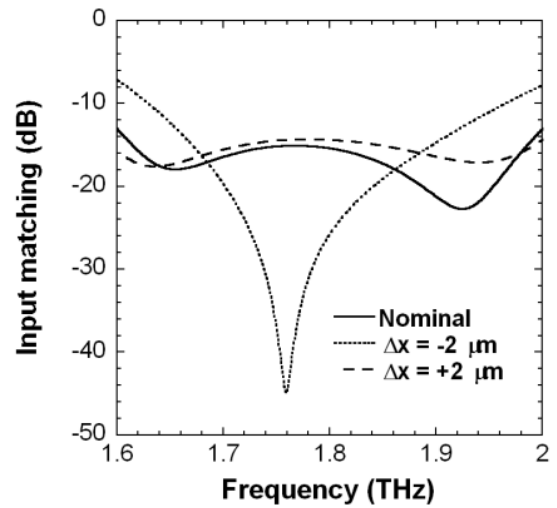

(b)

Fig. 13 Simulation of misalignment induced variations on the input matching. The position of the Si beam is varied from its nominal position inside the channel along the $\mathrm{X}$ and $\mathrm{Y}$-axis by $\pm 2 \mu \mathrm{m}$, according to the coordinate system presented in Fig.5.

\section{CONCLUSION}

In this paper, we presented our studies on the technology demonstrator for a balanced waveguide hot-electron bolometer mixer operating in the 1.6 $2.0 \mathrm{THz}$ band. The design uses a novel layout for the HEB mixer employing supporting $\Pi$-frame and combines several key technologies: all-metal $\mathrm{THz}$ waveguide micromachining, ultra-thin $\mathrm{NbN}$ deposition and a micromachining of silicon-on-insulator (SOI) substrate to manufacture the HEB mixer. We successfully completed all technological steps and final integration of the mixer. We believe that the demonstrated approach is suitable for building a single-end deep-terahertz mixer operating at up to $5 \mathrm{THz}$. The confirmed ease of integration by means of self-aligning of the mixer chip in the mixer housing opens prospective for making moderately large heterodyne terahertz array receivers. The proposed technology does not limit the number of pixels, which is rather constrained by a possibility to generate enough LO power. Even though demonstrated for HEB, the technology is suitable for other active mixer components such as Schottky diode- and SIS mixers or terahertz multipliers. In the near future, we plan to perform RF characterization including receiver noise measurements, IF bandwidth determination and investigate reproducibility and RF performance similarity of paired mixer chips.

\section{ACKNOWLEDGEMENTS}

Erik Sundin (GARD) is acknowledged for his help during the DC characterization. This work was supported by the European Commission 
Framework Programme 7, Advanced Radio Astronomy in Europe RadioNet, via JRA AMSTAR+, Grant No. 227290.

\section{REFERENCES}

[1] SOFIA: Stratospheric Observatory for Infrared Astronomy, http://www.sofia.usra.edu/index.html.

[2] Herschel Observatory, http://herschel.esac.esa.int/.

[3] Plank Observatory, http://www.esa.int/esaMI/Planck/index.html.

[4] N. Schneider, V. Minier, G. Durand, P. Tremblin, J. Urban, and P. Baron, "Atmospheric Transmission at Dome $\mathrm{C}$ between 0 and $10 \mathrm{THz}$," EAS Publications Series, vol. 40, pp. 327-332, 2010.

[5] D. Meledin, A. Pavolotsky, V. Desmaris, I. Lapkin, C. Risacher, V. P. Robles, D. Henke, O. Nyström, E. Sundin, D. Dochev, M. Pantaleev, M. Fredrixon, M. Strandberg, B. Voronov, G. Gol'tsman, and V. Belitsky, "A 1.3 THz balanced waveguide HEB mixer for the APEX telescope," IEEE Trans. Microwave Theory Tech., vol. 57, pp. 89-98, 2009.

[6] J. R. Pardo, E. Serabyn, and J. Cernicharo, "Submillimeter atmospheric transmission measurements on Mauna Kea during extremely dry El Nino conditions: implications for broadband opacity contributions," $J$. Quant. Spectrosc. Radiat. Transf., vol. 68, pp. 419-433, 2001.

[7] S. Paine, R. Blundell, D. C. Papa, and J. W. Barrett, "A Fourier transform spectrometer for measurement of atmospheric transmission at submillimeter wavelengths," PASP, vol. 112, pp. 108-118, 2000.

[8] D. P. Marrone, R. Blundell, E. Tong, S. N. Paine, D. Loudkov, J. H. Kawamura, D. Lühr, and C. Barrientos, "Observations in the 1.3 and $1.5 \mathrm{THz}$ atmospheric windows with the receiver lab telescope," in $16^{\text {th }}$ Int. Symp. Space Terahertz Technol. Göteborg, Sweden, 2005.

[9] G. de Lange, "Development of the HIFI band 3 and 4 mixer units," Proc. SPIE Int. Soc. Opt. Eng., vol. 5498, pp. 267-277, 2004.

[10] G. H. Tan, "The ALMA front ends: an overview," in Proc. $19^{\text {th }}$ Int. Symp.Space Terahertz Technol. Groningen, 2008.

[11] V. Belitsky, I. Lapkin, V. Vassilev, R. Monje, A. B. Pavolotsky, D. Meledin, D. Henke, O. Nyström, V. Desmaris, C. Risacher, M. Svensson, M. Olberg, E. Sundin, M. Fredrixon, D. Dochev, S.-E. Ferm, and H. Olofsson, "Facility Heterodyne Receiver for the Atacama Pathfinder Experiment Telescope," in Joint 32 ${ }^{\text {nd }}$ Int. Conf. Infrared Millimeter Waves and $15^{\text {th }}$ Int. Conf. Terahertz Electronics Cardiff, UK, 2007.

[12] P. Khosropanah, J. R. Gao, W. M. Laauwen, M. Hajenius, and T. M. Klapwijk, "Low noise NbN hot electron bolometer mixer at $4.3 \mathrm{THz}$," Appl. Phys. Lett., vol. 91, p. 221111, 2007. 
[13] S. Cherednichenko, V. Drankinskiy, T. Berg, P. Khosropanah, and E. Kollberg, "Hot-electron bolometer terahertz mixers for the Hershel Space Observatory," Rev. Sci. Inst., vol. 79, p. 034501, 2008.

[14] E. Tong, J. Kawamura, D. Marrone, D. Loudkov, S. Paine, R. Blundell, C. Barrientos, and D. Lühr, "A $1.5 \mathrm{THz}$ hot electron bolometer receiver for ground-based terahertz astronomy in northern Chile," Proc. SPIE, vol. 6373, 2006.

[15] J. W. Kooi and V. Ossenkopf, "HIFI instrument stability as measured during the thermal vacuum tests of the Herschel space observatory," Proc. $20^{\text {th }}$ Int. Symp. Space THzTechnol., 2009.

[16] A. Pavolotsky, D. Meledin, C. Risacher, M. Pantaleev, and V. Belitsky, "Micromachining approach in fabricating of $\mathrm{THz}$ waveguide components," Microelectron. J., vol. 36, p. 636, 2005.

[17] V. Desmaris, D. Meledin, A. Pavolotsky, R. Monje, and V. Belitsky, "All-metal micromachining for the fabrication of sub-millimetre and THz waveguide components and circuits," J. Micromech. Microeng., vol. 18, p. 095994, 2008.

[18] D. M. Pozar, Microwave engineering, 3rd ed.: John Wiley \& Sons, Inc., 2005.

[19] G. L. Matthaei, L. Young, and E. M. T. Jones, Microwave filters, impedance-matching networks, and coupling structures. Dedham Artech House, 1980.

[20] CST AG., CST Studio Suite 2009.

[21] Agilent Technologies, EMDS, 2000.

[22] G. Yassin and S. Withington, "Analytical expression for the input impedance of a microstrip probe in waveguide," Int. J. Infrared Millimeter Waves, vol. 17, pp. 1685-1705, 1996.

[23] J. Kooi, "A full-heigth waveguide to thin-film microstrip with exceptional RF bandwidth and coupling efficiency," Int. J. Infrared Millimeter Waves, vol. 24, pp. 261-283, 2003.

[24] C. Risacher, V. Vassilev, V. Belitsky, and A. Pavolotsky, "Waveguideto-microstrip transition with integrated bias - T," IEEE Microwave Wireless Comp. Lett., vol. 13, pp. 262 - 2642003.

[25] C. Risacher, V. Belitsky, V. Vassilev, I. Lapkin, and A. Pavolotsky, "A 275-370 GHz receiver SIS Mixer with novel probe structure," Int. J. Infrared and Millimeter Waves, vol. 26, pp. 867-879, 2005.

[26] S. Cherednichenko, V. Drakinskiy, J. Baubert, B. Lecomte, F. Dauplay, J.-M. Krieg, Y. Delorme, A. Feret, H.-W. Hübers, A. D. Semenov, and G. N. Gol'tsman, "2.5 THz multipixel heterodyne receiver based on NbN HEB mixers," Proc. SPIE, vol. 6275, 2006.

[27] R. B. Bass, J. C. Schultz, A. W. Lichtenberger, R. M. Weikle, S.-K. Pan, E. Bryerton, C. K. Walker, and J. W. Kooi, "Ultra-thin silicon 
chips for submillimeter-wave applications," in Proc. 15 th Int. Symp. Space \& THz Tech., Northampton, MA, USA, 2003, pp. 499-501.

[28] M. P. Lepselter, "Beam-Lead Technology," Bell. Syst. Tech. J., vol. 45, pp. 233-253, 1966.

[29] R. B. Bass, J. C. Schultz, A. W. Lichtenberger, J. W. Kooi, and C. K. Walker, "Beam lead fabrication for submillimeter-wave circuits using vacuum planarization," in Proc. $14^{\text {th }}$ Int. Symp. Space \& THz Tech., Tucson, AZ, USA, 2003, pp. 499-501.

[30] A. B. Kaul, B. Bumble, K. A. Lee, H. G. LeDuc, F. Rice, and J. Zmuidzinas, "Fabrication of wide-IF 200-300 GHz superconductorinsulator-superconductor mixers with suspended metal beam leads on silicon-on-insulator," J. Vac. Sci. Technol. B, vol. 22, pp. 2417-2422, 2004.

[31] P. H. Siegel, R. P. Smith, M. C. Gaidis, and S. C. Martin, "2.5-THz GaAs monolithic membrane-diode mixer," IEEE Trans. Microwave Theory Tech., vol. 47, pp. 596-604, 1999.

[32] J. Kooi, C. D. d'Aubigny, R. B. Bass, C. Walker, and A. W. Lichtenberger, "Large RF bandwidth waveguide to thinfilm microstrip transitions on suspended membrane for use in silicon micromachined mixer blocks at THz frequencies," in Proc. 14 ${ }^{\text {th }}$ Int. Symp. Space $\mathrm{THz}$ Tech., Tucson, AZ, 2003.

[33] ANSYS Inc., 275 Technology Drive, Canonsburg, PA 15317, USA.

[34] D. Roundy and M. L. Cohen, "Ideal strength of diamond, Si, and Ge," Phys. Rev. B, vol. 64, pp. 212103(1)-212103(3), 2001.

[35] Ultrasil Corporation, 3527 Breakwater Ave., Hayward, CA 94545, USA.

[36] D. Dochev, V. Desmaris, A. Pavolotsky, D. Meledin, Z. Lai, A. Henry, E. Janzén, E. Pippel, J. Woltersdorf, and V. Belitsky, "Growth and characterization of epitaxial ultra-thin $\mathrm{NbN}$ films on 3C-SiC/Si substrate for terahertz applications," Supercond. Sci. Technol., vol. 24, pp. 035016(1)-035016(6), 2011.

[37] F. Laermer and A. Schilp, "Method of anisotropically etching silicon," U.S. Patent No. 5501893, 1996. 